\title{
Kelvin probe study of the gas sensing properties of porphyrins-coated ZnO nanorods
}

\author{
Y. Sivalingam ${ }^{1}$, G. Magna ${ }^{1}$, E. Martinelli ${ }^{1}$, G. Pomarico ${ }^{2}$, A. Catini ${ }^{1}$, \\ A. D'Amico ${ }^{1}$, R. Paolesse ${ }^{2}$, C. Di Natale ${ }^{1}$ \\ ${ }^{1}$ Department of Electronic Engineering, University of Rome Tor Vergata, Via del Politecnico 1, 00133 \\ Roma, Italy \\ dinatale@uniroma2.it \\ ${ }^{2}$ Department of Chemical Science and Technology, University of Rome Tor Vergata, Via della Ricerca \\ Scientifica, 00133 Roma, Italy
}

\begin{abstract}
Hybrid materials formed by layers of porphyrins onto $\mathrm{ZnO}$ surface show an interesting combination of photonic and gas sensing properties. In this paper, we investigate, with the Kelvin probe technique, the changes of the surface potential of porphyrins coated $\mathrm{ZnO}$ nanowires induced by concurrent adsorption of gas and visible light illumination.
\end{abstract}

Key words: porphyrins, $\mathrm{ZnO}$ nanorods, Kelvin Probe, Volatile Compounds

\section{Introduction}

Porphyrins and $\mathrm{ZnO}$ are, in their respective realms, excellent materials for chemical sensors. Furthermore their combination gives rise to properties such as enhanced photovoltage [1] and improved catalytic efficiency [2]. The combination of these attributes is also supposed to improve the chemical sensitivity. To this regard, previous observations shown the great influence of the exposure to donor compounds (such as amines) on the conductivity of porphyrins coated $\mathrm{ZnO}$. Interestingly, since the sensitivity is triggered by the illumination to visible light, the sensor may be photo-addressable [3].

Besides than conductivity, other measurable quantities are expected to show a similar chemical sensitivity. Among them, the surface potential is particularly interesting being related to ChemFET devices. Here we studied the combined effect of light and gas on porphyrinscoated $\mathrm{ZnO}$ nanorods. The surface potential was investigated with the Kelvin probe technique.

\section{Experimental}

$\mathrm{ZnO}$ nanorods were grown by hydrothermal method onto ITO substrate previously coated with a seed layer [4]. The seed layer solution was formed by zinc acetate dehydrate dissolved at the concentration of $0.0275 \mathrm{~g}$ in 25 $\mathrm{mL}$ ethylene glycol.

Seed layer was properly patterned in order to obtain selected spots of nanorod arrays onto the substrate. For the scope an automatic dispenser (MicroDrop Gmbh) with $70 \mu \mathrm{m}$ nozzle was utilized.

In order to match the Kelvin probe size, spots diameter had to be larger than $3 \mathrm{~mm}$; about 30,000 droplets were necessary to cover the requested area.

After seeding processes, the samples were immersed in $25 \mathrm{mM}$ zinc acetate and hexamethylenetetramine solution for 4 hours in order to grow $\mathrm{ZnO}$ nanowires by hydrothermal method.

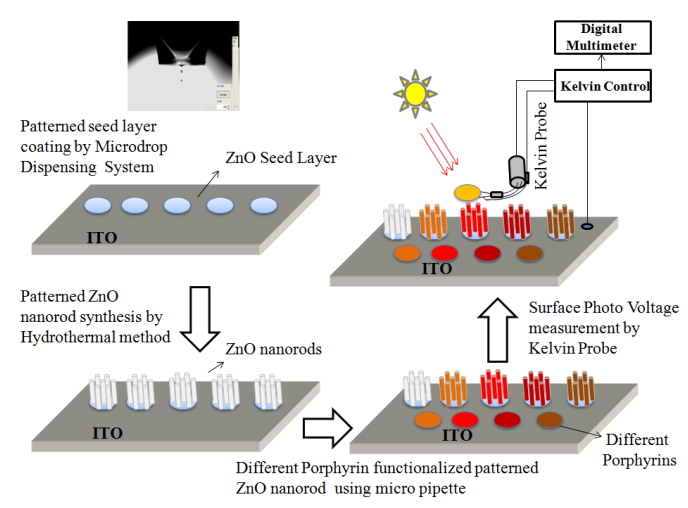

Fig. 1. Schematic view of the sensing materials fabrication and the measurement setup. 
After the synthesis the sample was characterized by scanning electron microscope (SEM) (Cambridge 360 Microscope (LaB6), U.K) to ascertain crystallinity and morphology. Figure 1 shows the experimental setup and the conceptual steps necessary for the fabrication of the sensitive materials. Hydrothermal technique was used to grow vertically aligned $\mathrm{ZnO}$ nanorods. Figure 2 shows a SEM image of the nanorods appearance.

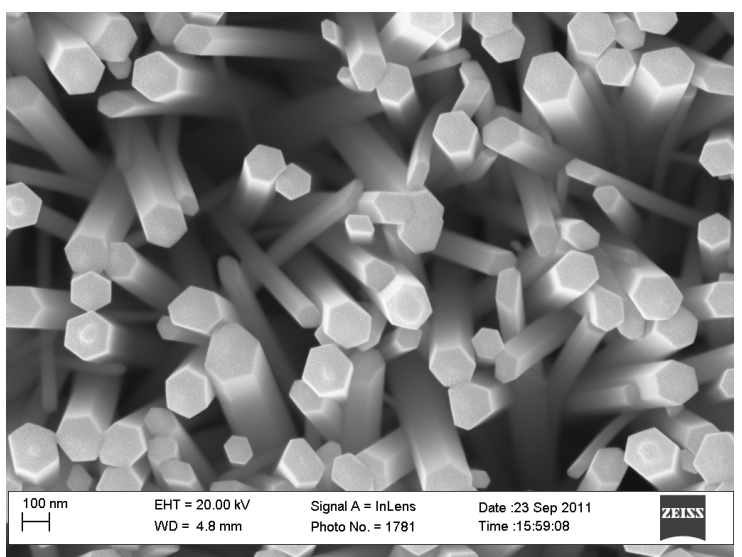

Fig. 2. Top view SEM image of the $\mathrm{ZnO}$ nanorods forest.

A Zinc Tetraphenylporphyrin (ZnTPP) was chosen as a model coating. This porphyrin has been frequently used for sensors purposes and it also shows brilliant optical properties [5]. To study the influence of the interaction between porphyrins and $\mathrm{ZnO}$ surface, the ZnTPP has been decorated with two alternative functional groups: either one carboxylic acid $(\mathrm{COOH})$ or four sulfonate groups $\left(\mathrm{SO}_{3} \mathrm{H}\right)$. Hence two materials were formed based on $\mathrm{ZnO}$ nanorods coated by $\mathrm{ZnTPPCOOH}$ and $\mathrm{ZnTPPSO}_{3} \mathrm{H}$. Molecular structures are visible in Figure 3.

Contact potential difference (CPD) has been measured with a commercial Kelvin probe equipment provided by Besocke Gmbh [6]. It consists in a gold pad of $3 \mathrm{~mm}$ diameter actuated by a piezoelectric element at a swinging frequency of $180 \mathrm{~Hz}$. The Kelvin probe is driven by an electronic system allowing for an automatic measurement of the contact potential difference. Surface photovoltage was measured illuminating the device with a white LED whose spectral features cover the absorbance spectra of porphyrins.

Chemical sensitivity was tested exposing the sensing layer to vapours of alcohols (ethanol and butanol) and amines (trimethylamine and triethylamine) diluted in nitrogen gas. In case of ethanol, butanol and triethylamine saturated vapors (at the temperature of $25^{\circ} \mathrm{C}$ ) were mixed to the nitrogen carrier gas, while for trimethylamine was used a certified tank, then diluted with nitrogen. The dilutions were kept constant by a computer controlled system of mass flow controllers.
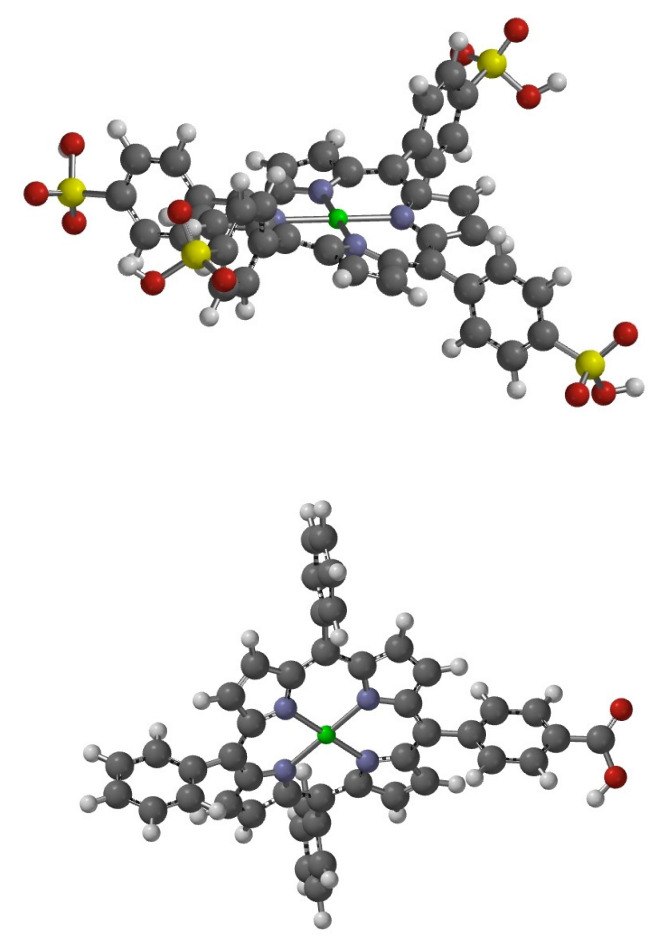

Fig. 3. Molecular structure of the studied ZnTPP. top) ZnTPPSO ${ }_{3} \mathrm{H}$, bottom) ZnTPPCOOH.

\section{Results and discussion}

Solid-state layers of porphyrins lying on different substrates exhibit surface photovoltage. The magnitude of the photovoltage may depend on the interactions between the porphyrin and the substrate. Here the surface photovoltage of $\mathrm{ZnTPPCOOH}$ and $\mathrm{ZnTPPSO}_{3} \mathrm{H}$ on $\mathrm{ZnO}$ are different from the values measured in ITO. The shift of CPD between dark and light is barely similar for both the porphyrins: $-142 \mathrm{mV}$ and $-130 \mathrm{mV}$ for $\mathrm{ZnTPPSO} \mathrm{H}_{3} \mathrm{H}$ and $\mathrm{ZnTPPCOOH}$ respectively.

In this paper we were interested in studying how the exposure to vapors alters the photovoltage. In practice, the changes of CPD due to exposure to volatile compounds have been measured in dark and in light conditions. Two alcohols: ethanol (EtOH) and butanol $(\mathrm{BuOH})$ and two amines: trimethylamine $\left(\mathrm{Me}_{3} \mathrm{~N}\right)$ and triethylamine $\left(\mathrm{Et}_{3} \mathrm{~N}\right)$ have been tested here. These compounds are good combinations of coordination and van der Waals interactions that are supposed to bind volatile compounds onto ZnTPP layers. Figure 4 shows a typical measurement session. CPD 
is continuously recorded using the automatic CPD measurement set-up included in the "Kelvin control 07" unit provided by Besocke Gmbh.

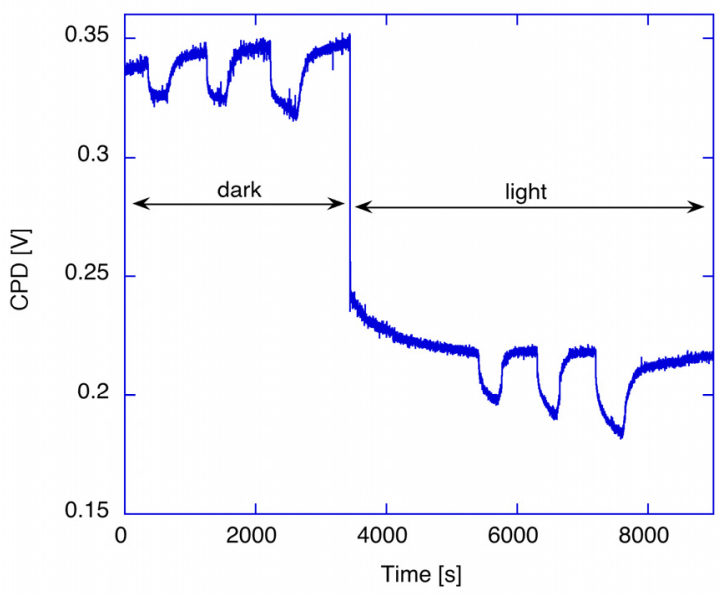

Fig. 4. Continuos recording of the $C P D$ in $\mathrm{ZnTPPCOOH}$ coated $\mathrm{ZnO}$. The illumination induces a shift of the CPD of about $-130 \mathrm{mV}$.Both in dark and in light, the sensing layer is exposed to three pulses of ethanol diluted in nitrogen gas. The concentration corresponded to $10 \%, 20 \%$ and $30 \%$ of the saturated pressure in a total pressure of $1 \mathrm{~atm}$.

As expected, amines elicit larger CPD changes than alcohols. On the other hand, the behavior under illumination is rather different for the two molecular configurations. Figures 5 and 6 show the magnitude of the COD change observed in dark and in light conditions for the two molecular systems exposed to various concentrations of the four test compounds. Results of figures 5 and 6 show that the functionalization plays a fundamental role in determining the sensitivity of the CPD in dark and in light to the volatile compounds. The signal for the $\mathrm{COOH}$ functionalization is generally larger with respect to that shown by porphyrins functionalized with the $\mathrm{SO}_{3} \mathrm{H}$ group. The most remarkable differences are found in the effect of the light to the sensor signals. In the case of the $\mathrm{SO}_{3} \mathrm{H}$ functional group an increase of the signal is observed for the amines while for alcohols the signals, more limited in intensity, are barely similar. In the case of the $\mathrm{COOH}$ functional group a clear increase of signals is observed for ethanol and triethylamine while in case of butanol, and more dramatically, for triethylamine the signals in dark largely exceed those recorded in light conditions.

A more complete view of the sensors behavior can be achieved treating the sensors data with the principal component analysis (PCA). PCA results are summarized in Figure 7 as a biplot where the scores (the samples) and the loadings (the sensors) are simultaneously plotted. The different signal intensities for amines and alcohols result in the net separation, along PC1, of amines and alcohols. More interesting is the separation among PC2 of lighter alcohols and amines $(\mathrm{EtOH}$ and $\left.\mathrm{Me}_{3} \mathrm{~N}\right)$ with respect to heavier molecules $\left(\mathrm{Et}_{3} \mathrm{~N}\right.$ and $\mathrm{BuOH}$ ). In terms of chemical interactions, the differences along PC2 seem to be ruled by the sorption interactions that are much more effective for butanol and triethylamine with respect to ethanol and trimethylamine. These interactions are quantified by the Ostwald parameter $\left(\log \mathrm{L}^{16}\right)$ appearing in the LSER interaction model [7]. For the couple of alcohols, this parameter is 1.485 for $\mathrm{EtOH}$ and 2.601 for $\mathrm{BuOH}$. For the amines, the sorption parameter is 1.62 for $\mathrm{Me}_{3} \mathrm{~N}$ and 3.04 for $\mathrm{Et}_{3} \mathrm{~N}$.

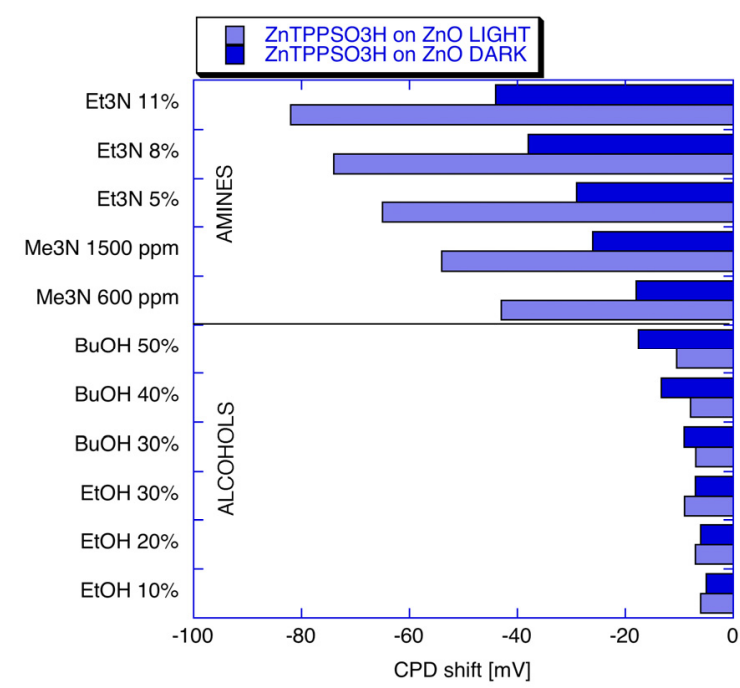

Fig. $5 \mathrm{CPD}$ signals shift for $\mathrm{ZnTPPSO}_{3} \mathrm{H}$

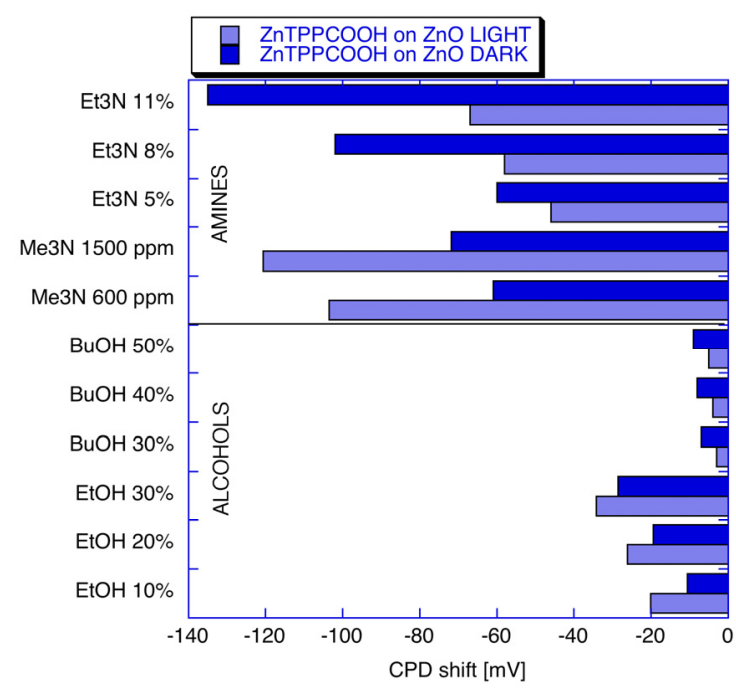

Fig. 6 CPD signal shifts for ZnTPPCOOH.

While all the loadings are equally oriented along $\mathrm{PC} 1$, along $\mathrm{PC} 2 \mathrm{SO}_{3} \mathrm{H}$ are oriented towards 
$\mathrm{BuOH}$ and $\mathrm{Et}_{3} \mathrm{~N}$ while $\mathrm{COOH}$ are colinear with $\mathrm{Me}_{3} \mathrm{~N}$ and $\mathrm{EtOH}$. In both cases the transition from dark to light does not influence drastically the correlation of the respective loadings with scores.

Clearly, the different behavior of the sensing layer is due to the spatial arrangement of the molecule over the $\mathrm{ZnO}$ surface. As a consequence it displays the influence of the functional group in the molecular organization at the $\mathrm{ZnO}$ surface. $\mathrm{SO}_{3} \mathrm{H}$ seems then to favour the sorption interaction while with the $\mathrm{COOH}$ group the specific interactions (likely the coordination to the zinc ion) prevail over the unspecific sorption forces.

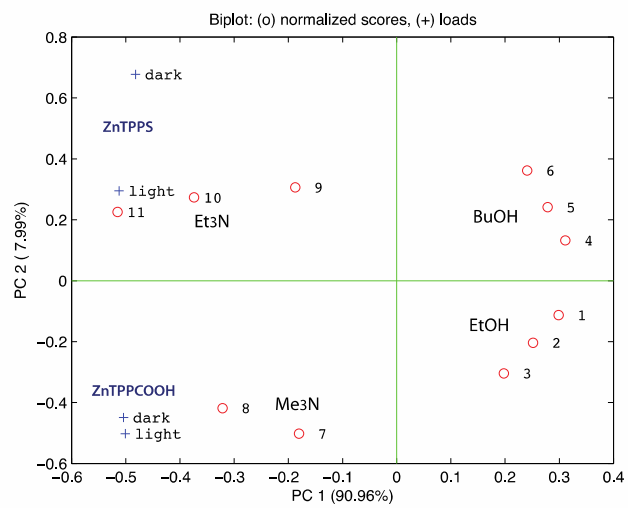

Fig. 7 Biplot of the PCA of the matrix collecting together the signals of the sensors in both light and dark conditions. Scores are plotted as red circles and loadings as blue crosses.

\section{Conclusions}

The interaction between substrate and porphyrins has not been sufficiently considered in particular from the point of view of the sensing properties. Here the important combination of porphyrins and a metal-oxide surface has been studied with the Kelvin probe method. Results indicate that the surface potential of porphryins is sensitive to the adsorption of alcohols and amines and furthermore the illumination with visible light may alter the sensitive behavior, and positive and negative effects may also be observed for different volatile compounds. This paper evidences that an important role is played by the properties of the functional group supposed to anchor the porphyrin to the $\mathrm{ZnO}$ surface. In particular, these results suggest that sulfonate group tend to promote the unspecific Van der Walls interactions while carboxyl group tends to favour the specific interaction of coordination. This different behaviour can be explained either with a spatial organization of the molecules onto to metal-oxide surface or with a diverse efficiency of anchoring interaction. Further investigations will be necessary to deeply understand the behaviour, nonetheless the interaction between porphyrin and substrate offer a further degree of freedom for the design of porphyrin based chemical sensors.

\section{References}

[1] Q. Zhao, M. Yu, T. Xie, L. Peng, P. Wang, D. Wang, Photovoltaic properties of a $\mathrm{ZnO}$ nanorod array affected by ethanol and liquid-crystalline porphyrin, Nanotechnology, 19, 245706 (2008)doi:10.1088/0957-4484/19/24/245706

[2] X. Li, Y: Cheng, S. Kang, J. Mu, Preparation and enhanced visible light driven catalytic activity of $\mathrm{ZnO}$ nanorods sensitized by porphyrin heteroaggregate, Applied Surface Science, 256, 6705-6709

(2010)doi:10.1016/j.apsusc.2010.04.074.

[3] Y. Sivalingam, E. Martinelli, A. Catini, G. Magna, G. Pomarico, R. Paolesse, A. D'Amico, C. Di Natale, Monocarboxy Tetraphenylporphyrin functionalized $\mathrm{ZnO}$ nanorods photoactivated gas sensor, Procedia Engineering 25, 1333-1336 (2011)doi:10.1016/j.proeng.2011.12.329.

[4] L. Greene, M. Law, D. Tan, M. Montano, J. Goldberger, G. Somorjai, P. Yang, General route to vertical $\mathrm{ZnO}$ nanowire arrays using textured ZnO seeds, Nano Letters 5, 1231-1236 (2005) doi: 10.1021/nl050788p.

[5] D. Filippini, A. Alimelli, C. Di Natale, R. Paolesse, A. D'Amico, I. Lundstrom, Chemical sensing with familiar devices, Angew Chemie Int Ed. 45, 38003803 (2006) doi: DOI : 10.1002/anie.200600050.

[6] K. Besocke, S. Berger, Piezoelectric driven Kelvin probe for contact potential difference studies, Rev Sci Instruments 47, 840-842 (1976) doi: $10.1063 / 1.1134750$. 